\title{
MEASURABLE FUNCTIONS AND SPHERICAL SUMMABILITY OF MULTIPLE FOURIER SERIES $\left({ }^{1}\right)$
}

\author{
BY \\ ROGER COOKE
}

I. Introduction. This paper is concerned with two problems on the structure of measurable functions which have been completely solved for functions of one variable but only partly solved for several variables. Theorems 1 and 2 below illustrate the usefulness of spherical summability of Fourier series of almost periodic functions and show that much of what was obtained for one variable by complicated and difficult constructions is given in greater generality by using an important result on spherical summability. For the history of the problems considered and some surprising applications of the results, cf. [3], [4], and [1].

II. Statement of the results. The results of Theorems 1 and 2 can be proved for functions with values in an arbitrary Banach space. To avoid confusion the results will be stated for real-valued functions; and the proof of Theorem 1 will be given only for two variables, i.e., for the square $J^{2},\left(J^{2}=[-\pi, \pi] \times[-\pi, \pi], J^{k}\right.$ is the product of $k$ copies of the interval $[-\pi, \pi])$.

THEOREM 1. Let $f\left(x_{1}, \ldots, x_{k}\right)$ be any function on $J^{k}$ which is measurable and finite valued almost everywhere. There exists a continuous additive interval function $F(I)$ such that almost everywhere $F^{\prime}\left(x_{1}, \ldots, x_{k}\right)=f\left(x_{1}, \ldots, x_{k}\right)$ and a trigonometric series $\sum a_{n_{1}, n_{k}} \exp \left[\left(n_{1} x_{1}+\cdots+n_{k} x_{k}\right)\right]$ which is summable with sum $f\left(x_{1}, \ldots, x_{k}\right)$ almost everywhere by means of any summation function of type $(k, k)$.

REMARKS. For the notation $F^{\prime}\left(x_{1}, \ldots, x_{k}\right)$ and all elementary concepts about interval functions, cf. Saks [5]. The first half of this theorem was stated by Saks. What is original in the present paper is the preliminary results (Lemmas 2 and 3); the emphasis is on the second half of the theorem, which seems to require a localization principle for its proof both in one variable and here. For definitions and facts about summation functions, cf. [2].

THEOREM 2. Let $f(x)$ be any function on the reals which is measurable and finite valued almost everywhere. There exists a continuous almost periodic function $F(x)$ such that $F^{\prime}(x)=f(x)$ almost everywhere and a trigonometric series $\sum c_{\lambda} e^{i \lambda x}$ summable with sum $f(x)$ almost everywhere by means of any summation function of type $(1,1)$.

Received by the editors November 15, 1966.

(1) These results form part of the author's doctoral dissertation. I am grateful to my advisor, Professor Bochner, for many helpful conversations. This work was done while the author held a National Science Foundation Graduate Fellowship. 
III. Proof of the theorems. The notation follows Saks [5], except for the strong derivative $F_{s}^{\prime}$ which we denote by $F^{*}$.

LEMMA 1. Let $c>0$. For every $\varepsilon>0$ there exists $\delta>0$ such that $d(I)<\varepsilon$ for any interval I such that $m(I)<\delta$ and $r(I) \geqq 2 c$.

Proof. Let $K$ be a square containing the interval $I$ such that $m(I) \geqq m(K) c\left(^{2}\right)$. If $e$ is the length of an edge of $K$, then $m(K)=e^{2}$ and $d(K)=e \sqrt{ } 2$. The lemma now follows from the relations

$$
0 \leqq d(I) \leqq d(K)=\sqrt{ } 2[m(K)]^{1 / 2} \leqq \sqrt{ } 2(1 / c)^{1 / 2}[m(I)]^{1 / 2}
$$

Next we need an approximation theorem for additive interval functions which requires a somewhat artificial correspondence between interval functions and point functions on the square $J^{2}$. The correspondence is as follows: an interval function $F(I)$ defines a function $F(x, y)$ by

$$
F(x, y)=F\left(I_{(x, y)}\right)
$$

where $I_{(x, y)}$ is the rectangle whose vertices are $(-\pi,-\pi),(-\pi, y),(x,-\pi),(x, y)$. It is easy to show that the correspondence (1) is one-to-one between continuous additive interval functions $F(I)$ and continuous functions $F(x, y)$ such that $F(s, t)=0$ if either $s=-\pi$ or $t=-\pi$. In fact if one defines an additive interval function $F(I)$ corresponding to the point function $F(x, y)$ by

$$
F([a, b] \times[c, d])=F(b, d)-F(a, d)-F(b, c)+F(a, c)
$$

it is clear that (1) and (2) are inverses of each other for the classes mentioned, and if one defines $|F(I)|_{\infty}=\sup _{I}|F(I)|$, equations (1) and (2) imply

$$
\|F(x, y)\|_{\infty} \leqq|F(I)|_{\infty} \leqq 4\|F(x, y)\|_{\infty} .
$$

In particular, the continuous additive interval functions form a Banach space $\mathscr{T}\left(J^{2}\right)$ under this norm, and this space is isomorphic to the Banach algebra $C_{0}(X)$ of continuous functions vanishing at infinity on a locally compact Hausdorff space $X$ (namely $J^{2}$ minus its lower edges).

From this point on a continuous additive interval function will be called a caif.

LEMMA 2. Continuous additive interval functions $F(I)$ such that $F^{*}(x, y)=0$ almost everywhere are dense in the space $\mathscr{T}\left(J^{2}\right)$.

Proof. Continuous functions of the form $\sum_{i=1}^{n} f_{i}(x) g_{i}(y)$ with $f_{i}^{\prime}(x)=g_{i}^{\prime}(x)=0$ a.e. on $(-\pi, \pi]$ form a subalgebra of $C_{0}(X)$. This subalgebra separates points and for each point $(x, y)$ contains a function which does not vanish there. By the StoneWeierstrass theorem these functions are dense in $C_{0}(X)$; the interval functions they generate are dense in $\mathscr{T}\left(J^{2}\right)$. It remains to be shown that these functions have strong derivative equal to zero almost everywhere.

(2) Although $r(I)$ is defined in [5] as $\inf _{I \subset_{X}} m(I) / m(K)$, it is obvious that $\sup _{I} \in_{X} m(I) / m(K)$ is what was intended. 
Since derivation is a linear operation, it is sufficient to show that $H^{*}(x, y)=0$ a.e. if $H(I) \sim f(x) g(y)$ and $f^{\prime}(x)=g^{\prime}(x)=0$ almost everywhere. Let $E=\left\{x: f^{\prime}(x)=0\right\}$, $F=\left\{y: g^{\prime}(y)=0\right\}$. The complement of $E \times F$ has measure zero.

Let $(x, y) \in E \times F$. There exists $M$ such that

$$
\left|\frac{f\left(x_{2}\right)-f\left(x_{1}\right)}{x_{2}-x_{1}}\right| \leqq M \text { if } x \in\left[x_{1}, x_{2}\right]
$$

and

$$
\left|\frac{g\left(y_{2}\right)-g\left(y_{1}\right)}{y_{2}-y_{1}}\right| \leqq M \text { if } y \in\left[y_{1}, y_{2}\right]
$$

Furthermore, for every $\varepsilon>0$ there exists $\delta>0$ such that

$$
\begin{aligned}
& \left|\frac{f\left(x_{2}\right)-f\left(x_{1}\right)}{x_{2}-x_{1}}\right| \leqq \frac{\varepsilon}{M} \quad \text { if } x \in\left[x_{1}, x_{2}\right] \text { and }\left|x_{2}-x_{1}\right|<\delta, \\
& \left|\frac{g\left(y_{2}\right)-g\left(y_{1}\right)}{y_{2}-y_{1}}\right| \leqq \frac{\varepsilon}{M} \text { if } y \in\left[y_{1}, y_{2}\right] \text { and }\left|y_{2}-y_{1}\right|<\delta
\end{aligned}
$$

Then if $I=\left[a_{1}, b_{1}\right] \times\left[a_{2}, b_{2}\right]$ and $m(I)<\delta^{2}$ we have either $\left|b_{1}-a_{1}\right|<\delta$ or $\left|b_{2}-a_{2}\right|<\delta$. In either case, if $(x, y) \in I$ we must have

$$
\left|\frac{H(I)}{m(I)}\right|=\left|\frac{f\left(b_{1}\right)-f\left(a_{1}\right)}{b_{1}-a_{1}}\right| \cdot\left|\frac{g\left(b_{2}\right)-g\left(a_{2}\right)}{b_{2}-a_{2}}\right|<\varepsilon .
$$

Therefore $H^{*}(x, y)=0$.

COROLlary. Let $F_{1}, F_{2}$ be continuous additive interval functions and suppose that $F_{2}$ has a strong [resp. ordinary] derivative almost everywhere. For any $\varepsilon>0$ there exists a continuous additive interval function $F$ such that $\left|F-F_{1}\right|_{\infty}<\varepsilon$ and $F^{*}=F_{2}^{*}$ [resp. $\left.F^{\prime}=F_{2}^{\prime}\right]$ almost everywhere.

Proof. By the lemma there exists a caif $G$ such that $G^{*}(x, y)=0$ almost everywhere and $\left|G-\left(F_{1}-F_{2}\right)\right|_{\infty}<\varepsilon$. Let $F=G+F_{2}$.

LEMMA 3. Let $g(x, y)$ be a bounded measurable function, $P$ a closed subset of $J^{2}$ and $\varepsilon>0$. There exists a caif $G$ such that

(1) $G^{\prime}(x, y)=0$ if $(x, y) \in P$;

(2) $G^{*}(x, y)=g(x, y)$ a.e. on $J^{2}-P$;

(3) $|G(I)| \leqq \varepsilon \cdot m(I)$ if $r(I) \geqq \varepsilon$ and $I \cap P \neq \varnothing$;

(4) $|G(I)| \leqq \varepsilon$ for all $I \subset J^{2}$;

(5) the function

$$
G^{(x, y)}(s, t)=\frac{G(s, t)-G(x, t)-G(s, y)+G(x, y)}{(s-x)^{2}+(t-y)^{2}}
$$

is in $L^{\infty}$ for almost every $(x, y)$ and, in particular, $\left\|G^{(x, y)}\right\|_{\infty} \leqq \varepsilon$ if $(x, y) \in P$. 
Proof. By Vitali's covering theorem, there exists a disjoint sequence $\left\{V_{n}\right\}_{n=1}^{\infty}$ of closed intervals $V_{n} \subset J^{2}-P$ such that $m\left(J^{2}-P-\bigcup_{n=1}^{\infty} V_{n}\right)=0$. In each $V_{n}$ there exists a caif $G_{n}$ such that $G_{n}^{*}(x, y)=g(x, y)$ a.e. and $\left|G_{n}\right|_{\infty}$ can be as small as desired (cf. [5, Theorem 10.7], and apply the corollary to Lemma 2).

Since $V_{n}$ and $P$ are disjoint compact sets, they lie at a positive distance $\rho_{n}$ from each other and there exists $\eta_{n}, 0<\eta_{n}<1$ such that $I \cap V_{n}=\varnothing$ if only $r(I) \geqq 2^{-n} \cdot \varepsilon$, $m(I) \leqq \eta_{n}$, and $I \cap P \neq \varnothing$ (cf. Lemma 1). Defining $G_{n}(\varnothing)=0$ and requiring

$$
\left|G_{n}\right|_{\infty} \leqq 2^{-n} \rho_{n}^{\prime} \cdot \eta_{n} \cdot \varepsilon
$$

where $\rho_{n}^{\prime}$ is the smaller of 1 and $\rho_{n}^{2}$ we have

$$
\left|G_{n}\left(I \cap V_{n}\right)\right| / m(I) \leqq 2^{-n} \cdot \varepsilon \text { if } r(I) \geqq 2^{-n} \cdot \varepsilon \text { and } I \cap P \neq \varnothing .
$$

For if $m(I) \leqq \eta_{n}, I \cap V_{n}=\varnothing$. Otherwise (4) follows from (3).

Having fixed a choice of $G_{n}$ we set $G(I)=\sum_{n=1}^{\infty} G_{n}\left(I \cap V_{n}\right)$. It is clear from (3) that the series converges uniformly. Thus $G$ is a caif. To verify assertion (1), let $I_{p} \rightarrow(x, y) \in P$ and $r\left(I_{p}\right) \geqq c>0$. Choose $n_{0}$ so large that $2^{-n_{0} \cdot \varepsilon<c}$. Then

$$
\frac{G\left(I_{p}\right)}{m\left(I_{p}\right)}=\sum_{n<n_{0}} \frac{G_{n}\left(I_{p} \cap V_{n}\right)}{m\left(I_{p}\right)}+\sum_{n \geqq n_{0}} \frac{G_{n}\left(I_{p} \cap V_{n}\right)}{m\left(I_{p}\right)} .
$$

If $n \geqq n_{0}$, then $\left|G_{n}\left(I_{p} \cap V_{n}\right)\right| / m\left(I_{p}\right) \leqq 2^{-n} \cdot \varepsilon$ for all $p$ by (4). Since $G_{n}\left(I_{p} \cap V_{n}\right) / m\left(I_{p}\right)$ $\rightarrow 0$ as $p \rightarrow \infty$ for all $n$, the dominated convergence theorem for series implies that $G^{\prime}(x, y)=0$.

To verify (2) we observe that since $V_{n}$ is an interval it has the same measure as its interior (int $V_{n}$ ). If $(x, y) \in \operatorname{int}\left(V_{n}\right) \cap I_{p}$ and $d\left(I_{p}\right)$ is small enough, $I_{p} \subset$ int $V_{n}$, so that $G\left(I_{p}\right) / m\left(I_{p}\right)=G_{n}\left(I_{p}\right) / m\left(I_{p}\right)$. Hence $G^{*}(x, y)=G_{n}^{*}(x, y)=g(x, y)$ a.e. on int $V_{n}$, and thus a.e. on $V_{n}$. Since the $V_{n}$ are disjoint $G^{*}(x, y)=g(x, y)$ a.e. on $\bigcup_{n=1}^{\infty} V_{n}$ and finally a.e. on $J^{2}-P$.

Assertion (3) is obvious, since if $r(I) \geqq \varepsilon$, then $r(I) \geqq \varepsilon \cdot 2^{-n}, n=1,2, \ldots$. Hence if $I \cap P \neq \varnothing$, then

$$
|G(I)| \leqq \sum_{n=1}^{\infty}\left|G_{n}\left(I \cap V_{n}\right)\right| \leqq \sum_{n=1}^{\infty} 2^{-n} \cdot \varepsilon m(I)=\varepsilon \cdot m(I)
$$

Assertion (4) is also obvious since $|G(I)| \leqq \sum_{n=1}^{\infty}\left|G_{n}\left(I \cap V_{n}\right)\right| \leqq \sum_{n=1}^{\infty} 2^{-n} \cdot \varepsilon=\varepsilon$.

To verify assertion (5), let $\left(x_{0}, y_{0}\right)$ be a point of $J^{2}-P$ where $G^{*}\left(x_{0}, y_{0}\right)=g\left(x_{0}, y_{0}\right)$.

$$
\begin{aligned}
\left|G^{\left(x_{0}, y_{0}\right)}(s, t)\right| & =\left|\frac{\left(s-x_{0}\right)\left(t-y_{0}\right)}{\left(s-x_{0}\right)^{2}+\left(t-y_{0}\right)^{2}}\right|\left|\frac{G(s, t)-G\left(x_{0}, t\right)-G\left(s, y_{0}\right)+G\left(x_{0}, y_{0}\right)}{\left(s-x_{0}\right)\left(t-y_{0}\right)}\right| \\
& \leqq \frac{1}{2}\left|\frac{G(s, t)-G\left(x_{0}, t\right)-G\left(s, y_{0}\right)+G\left(x_{0}, y_{0}\right)}{\left(s-x_{0}\right)\left(t-y_{0}\right)}\right| .
\end{aligned}
$$

The function $G^{\left(x_{0}, y_{0}\right)}$ is continuous, and hence bounded, on the set of $(s, t)$ such that either $\left|s-x_{0}\right| \geqq \delta$ or $\left|t-y_{0}\right| \geqq \delta$ for any $\delta>0$. But for $\delta$ sufficiently small, the 
right-hand side of (5) is bounded when $0<\left|s-x_{0}\right|<\delta$ and $0<\left|t-y_{0}\right|<\delta$, since $G^{*}\left(s_{0}, y_{0}\right)$ exists.

If $\left(x_{0}, y_{0}\right) \in P$, then for $s>x_{0}, t>y_{0}$

$$
\left|G^{\left(x_{0}, y_{0}\right)}(s, t)\right|=\frac{\left|G\left(\left[x_{0}, s\right] \times\left[y_{0}, t\right]\right)\right|}{\rho\left((s, t),\left(x_{0}, y_{0}\right)\right)^{2}}
$$

Now

$$
\left|G\left(\left[x_{0}, s\right] \times\left[y_{0}, t\right]\right)\right| \leqq \sum_{n=1}^{\infty}\left|G_{n}\left(V_{n} \cap\left[x_{0}, s\right] \times\left[y_{0}, t\right]\right)\right|
$$

and $V_{n} \cap\left[x_{0}, s\right] \times\left[y_{0}, t\right]=\varnothing$ unless $\rho\left((s, t),\left(x_{0}, y_{0}\right)\right)^{2} \geqq \rho_{n}^{\prime}$. Thus the summation in the right-hand side of (7) is extended only over those $n$ for which this condition is satisfied.

But from (3) these terms satisfy

$$
\frac{\left|G_{n}\left(V_{n} \cap\left[x_{0}, s\right] \times\left[y_{0}, t\right]\right)\right|}{\rho\left((s, t),\left(x_{0}, y_{0}\right)\right)^{2}}<2^{-n} \cdot \varepsilon .
$$

From (7) and (8) we have $\left|G^{\left(x_{0}, y_{0}\right)}(s, t)\right| \leqq \varepsilon$. The cases $s \leqq x_{0}$ or $t \leqq y_{0}$ are handled similarly. This completes the proof.

LEMMA 4. Let $f(x, y)$ be any function on $J^{2}$ which is measurable and finite valued almost everywhere. There exists a caif $F(I)$ such that $F^{\prime}(x, y)=f(x, y)$ a.e. on $J^{2}$ and

$$
F^{(x, y)}(s, t)=\frac{F(s, t)-F(x, t)-F(s, y)+F(x, y)}{(s-x)^{2}+(t-y)^{2}}
$$

is in $L^{\infty}$ for almost every $(x, y) \in J^{2}$.

Proof. We shall define a sequence of closed sets $P_{n}$ and a sequence of caif's $G_{n}$ such that if $Q_{n}=\bigcup_{k=0}^{n} P_{k}$, and $F_{n}=\sum_{k=0}^{n} G_{k}$, the following conditions will be satisfied:

(1) $G_{n}^{\prime}(x, y)=0$ on $Q_{n-1}$;

(2) $G_{n}^{\prime}(x, y)=f(x, y)-F_{n-1}^{\prime}(x, y)$ a.e. on $P_{n}$; i.e., $F_{n}^{\prime}(x, y)=f(x, y)$ a.e. on $Q_{n}$;

(3) $\left|G_{n}(I)\right| \leqq 2^{-n} m(I)$ if $r(I) \geqq 2^{-n}$ and $I \cap Q_{n-1} \neq \varnothing$;

(4) $\left|G_{n}(I)\right| \leqq 2^{-n}$ for all $I \subset J^{2}$;

(5) $m\left(J^{2}-Q_{n}\right)<1 / n$;

(6) the function

$$
G_{n}^{(x, y)}(s, t)=\frac{G_{n}(s, t)-G_{n}(x, t)-G_{n}(s, y)+G_{n}(x, y)}{(s-x)^{2}+(t-y)^{2}}
$$

is in $L^{\infty}$ for almost every $(x, y) \in J^{2}$ and $\left\|G_{n}^{(x, y)}\right\|_{\infty} \leqq 2^{-n}$ if $(x, y) \in Q_{n-1}$.

To do this set $G_{0}(I)=0, P_{0}=\varnothing$. Assertions (1)-(6) are trivial in this case. Now suppose we have chosen $G_{k}, P_{k}$ so as to have (1)-(6) for $k \leqq r$. Let $E_{r} \subset J^{2}-Q_{r}$ be such that $f$ and $F_{r}^{\prime}$ are bounded on $E_{r}$ and $m\left(J^{2}-Q_{r}-E_{r}\right)<1 /(r+1)$. Applying 
Lemma 3 with $\varepsilon=2^{-(r+1)}, g=\chi_{E_{r}} \cdot\left(f-F_{r}^{\prime}\right), P=Q_{r}$ we find a caif $G_{r+1}$ such that

(a) $G_{r+1}^{\prime}(x, y)=0$ on $Q_{r}$;

(b) $G_{r+1}^{*}(x, y)=\left(f-F_{r}^{\prime}\right)(x, y)$ a.e. on $E_{r}$;

(c) $G_{r+1}(I) \leqq 2^{-(r+1)} m(I)$ if $r(I) \geqq 2^{-(r+1)}$ and $I \cap Q_{r} \neq \varnothing$;

(d) $G_{r+1}(I) \leqq 2^{-(r+1)}$ for all $I \subset J^{2}$;

(e) $G_{r+1}^{(x, y)}(s, t)$ is in $L$ for almost every $(x, y)$ and $\left\|G_{r+1}^{(x, y)}\right\|_{\infty} \leqq 2^{-(r+1)}$ if $(x, y) \in Q_{r}$.

If $P_{r+1}$ is a closed subset of $E_{r}$ such that $m\left(J^{2}-Q_{r}-P_{r+1}\right)<1 /(r+1)$, we clearly have assertions (1)-(6) for $k=r+1$. Now let $Q=\lim _{n} Q_{n}=\bigcup_{k=0}^{\infty} P_{k}, F=\lim _{n} F_{n}$ $=\sum_{k=0}^{\infty} G_{k}$, and let $S_{n}$ be the subset of $J^{2}$ for which one of assertions (2) and (6) fails. We have $m\left(\bigcup_{k=0}^{\infty} S_{k}\right)=0$, so that $m\left(J^{2}-\left(Q-\bigcup_{k=0}^{\infty} S_{k}\right)\right)=0$.

By assertion (4) we see that $F(I)$ is a caif. We claim that if $(x, y) \in Q-\bigcup_{k=0}^{\infty} S_{k}$, then $F^{\prime}(x, y)=f(x, y)$ and $F^{(x, y)} \in L^{\infty}$.

In fact, if $I_{p} \rightarrow(x, y)$ and $r\left(I_{p}\right) \geqq c>0$, choose $n_{0}$ such that $n \geqq n_{0}$ implies $(x, y) \in Q_{n}-\bigcup_{k=0}^{\infty} S_{k}$ and $2^{-n}<c$. Then for $n>n_{0}$

so that

$$
\frac{F\left(I_{p}\right)}{m\left(I_{p}\right)}=\frac{F_{n}\left(I_{p}\right)}{m\left(I_{p}\right)}+\sum_{k>n} \frac{G_{k}\left(I_{p}\right)}{m\left(I_{p}\right)}
$$

$$
\begin{aligned}
\limsup _{p \rightarrow \infty}\left|\frac{F\left(I_{p}\right)}{m\left(I_{p}\right)}-f(x, y)\right| & \leqq \lim _{p \rightarrow \infty}\left|\frac{F_{n}\left(I_{p}\right)}{m\left(I_{p}\right)}-f(x, y)\right|+\sum_{k>n} \frac{\left|G_{k}\left(I_{p}\right)\right|}{m\left(I_{p}\right)} \\
& \leqq 0+\sum_{k>n} 2^{-k}=2^{-n} .
\end{aligned}
$$

Since $n$ is arbitrary, $F^{\prime}(x, y)=f(x, y)$.

Finally $F^{(x, y)}(s, t)=\left(\sum_{k \leq n_{0}}+\sum_{k>n_{0}}\right)\left(G_{k}^{(x, y)}(s, t)\right)$ so that

$$
\left\|F^{(x, y)}\right\|_{\infty} \leqq \sum_{k \leqq n_{0}}\left\|G_{k}^{(x, y)}\right\|_{\infty}+2^{-n_{0}+1} .
$$

Proof of Theorem 1. The existence of the primitive $F$ is explicitly stated and proved in the last lemma. Let $(x, y)$ be a point in the interior of $J^{2}$ for which $F^{\prime}(x, y)=f(x, y)$ and for which $F^{(x, y)}$ is in $L^{\infty}$. Then

is also in $L^{\infty}$. Let

$$
H^{(x, y)}(s, t)=F^{(x, y)}(s, t)-f(x, y) \frac{\sin (s-x) \sin (t-y)}{(s-x)^{2}+(t-y)^{2}}
$$

$$
\begin{aligned}
K^{(x, y)}(s, t) & =H^{(x, y)}(s, t)\left[(s-x)^{2}+(t-y)^{2}\right] \\
& =F(s, t)-F(x, t)-F(s, y)+F(x, y)-f(x, y) \sin (s-x) \sin (t-y) .
\end{aligned}
$$

We claim that $K^{(x, y)}(s, t)$ has a two-fold zero at $(x, y)$. This means, of course, that

$$
\lim _{r \rightarrow 0} \frac{1}{r^{2}} \iint_{C_{r}}\left|\frac{K^{(x, y)}(s, t)}{(s-x)^{2}+(t-y)^{2}}\right| d s d t=0
$$

where $C_{r}$ is the disk of radius $r$ about $(x, y)$. Define a set $U_{c}$ for $c>0$ by

$$
U_{c}=\left\{(s, t): c|s-x| \leqq|t-y| \leqq c^{-1}|s-x|\right\} .
$$




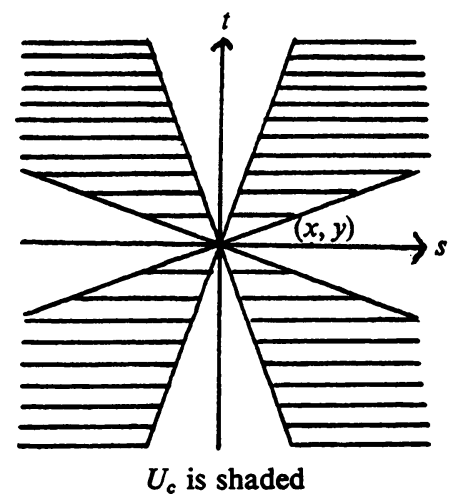

The integrand in (10) is just $H^{(x, y)}(s, t)$, which, as we remarked, is in $L^{\infty}$. For any fixed $c>0$ and $\varepsilon>0$ there exists $\delta>0$ such that $0<r<\delta$ and $(s, t) \in C_{r} \cap U_{c}$ imply $\left|H^{(x, y)}(s, t)\right|<\varepsilon$. This is because $F(I)$ has an ordinary derivative at $(x, y)$ equal to $f(x, y)$ so that

$$
\left|H^{(x, y)}(s, t)\right| \leqq\left|\frac{(s-x)(t-y)}{(s-x)^{2}+(t-y)^{2}}\right|
$$

$$
\begin{aligned}
& \cdot\left|\frac{F(s, t)-F(x, t)-F(s, y)+F(x, y)}{(s-x)(t-y)}-f(x, y) \frac{\sin (s-x)}{s-x} \frac{\sin (t-y)}{t-y}\right| \\
\leqq & \left|\frac{F(s, t)-F(x, t)-F(s, y)+F(x, y)}{(s-x)(t-y)}-f(x, y)\right| \\
& +|f(x, y)|\left|\frac{\sin (s-x) \sin (t-y)}{(s-x)(t-y)}-1\right|,
\end{aligned}
$$

and the right-hand side of (11) can be made less than $\varepsilon$ for all $(s, t) \in C_{r} \cap U_{c}$ by taking $r$ sufficiently small.

Now $m\left(C_{r}-U_{c}\right) / m\left(C_{r}\right)$ is a function of $c$ only; that is, $U_{c}$ occupies a fixed fraction of each disk with center at $(x, y)$. Furthermore this fraction tends to 1 as $c$ tends to 0 .

Hence we can choose $c>0$ such that $m\left(C_{r}-U_{c}\right) / m\left(C_{r}\right) \leqq \varepsilon / 2 \pi\left\|H^{(x, y)}\right\|_{\infty}$ for all $r>0$. For this $c$ and $\varepsilon$ choose $\delta$ such that $0<r<\delta$ and $(s, t) \in C_{r} \cap U_{c}$ implies $\left|H^{(x, y)}(s, t)\right|<\varepsilon / 2 \pi$.

Then if $0<r<\delta$, we have

$$
\begin{aligned}
0 & \leqq r^{-2} \iint_{C_{r}}\left|H^{(x, y)}(s, t)\right| d s d t \\
& \leqq r^{-2} \iint_{C_{r}-U_{c}}+r^{-2} \iint_{C_{r} \cap U_{c}} \\
& \leqq r^{-2} m\left(C_{r}-U_{c}\right)\left\|H^{(x, y)}\right\|_{\infty}+r^{-2} m\left(C_{r} \cap U_{c}\right) \cdot \varepsilon / 2 \pi \\
& \leqq r^{-2} m\left(C_{r}\right) \cdot \varepsilon / 2 \pi+r^{-2} m\left(C_{r}\right) \cdot \varepsilon / 2 \pi=\varepsilon / 2+\varepsilon / 2=\varepsilon .
\end{aligned}
$$

Since $\varepsilon$ is arbitrary, this establishes the assertion. 
Now by an established theorem [2, Theorem I] if $T$ is the Fourier series of $K^{(x, y)}$, and $D(x, y)=-x y$, then $D_{(s, t)}^{2} T_{R}^{\phi}(s, t) \rightarrow 0$ at $(x, y)$ for any summation function $\phi$ of type $(2,2)$.

If $S$ is the Fourier series of $F$, this means $D_{(s, t)}^{2} S_{R}^{\Phi}(s, t)-f(x, y) \rightarrow 0$ at $(s, t)=(x, y)$. Q.E.D.

The proof of Theorem 2 proceeds by means of another lemma. The first part of Theorem 1 assures the existence of a primitive, say $G(x)$, for any function $g(x)$, measurable and finite valued a.e. on any closed interval $[a, b]$. Lemma 2 implies that the sup-norm of $G$ can be arbitrarily small. Finally, by adding to $C$ a singular function of small norm we can also assume that $G(a)=G(b)=0$.

Thus we have the following result.

LEMMA 5. Let $g(x)$ be any function on the real line, measurable and finite valued a.e. For every $\varepsilon>0$ and every nonnegative integer $n$ there exists a continuous function $G(x)$ of period $2(n+1)$ satisfying the following conditions:

(1) $G(x)=0$ if $|x|<n$,

(2) $G^{\prime}(x)=g(x)$ a.e. on $n \leqq|x| \leqq n+1$,

(3) $\|G\|_{\infty} \leqq \varepsilon$.

Now we can define the function $F(x)$ whose existence is asserted in the theorem. We first let $F_{r}(x)$, for each nonnegative integer $r$ be a continuous function of period $2(r+1)$ such that

(a) $F_{r}(x)=0$ if $|x|<r$,

(b) $F_{r}^{\prime}(x)=f(x)-\left[F_{0}^{\prime}(x)+\cdots+F_{r-1}^{\prime}(x)\right]$ a.e. on $r \leqq|x| \leqq r+1$,

(c) $\left\|F_{r}\right\|_{\infty} \leqq 2^{-r}$.

We then set $F(x)=\sum_{r=0}^{\infty} F_{r}(x)$. It is clear that $F(x)$ is a limit periodic function, hence certainly almost periodic.

Now, almost everywhere on the set $n \leqq|x| \leqq n+1$, we have $F^{\prime}(x)=F_{0}^{\prime}(x)+\cdots$ $+F_{n}^{\prime}(x)=f(x)$ by (b). That is, $F$ is a primitive.

Now set $G^{(x)}(s)=F(s)-F(x)-f(x) \sin (s-x)$. Clearly $G^{(x)}(s)$ is an almost periodic function of $s$ for each $x$ where $F^{\prime}(x)=f(x)$ exists; that is, a.e. For such an $x, G^{(x)}(s)$ has a zero of order 1 at $x$, because

$$
\lim _{h \rightarrow 0} \frac{1}{h} \int_{x-h}^{x+h}\left|\frac{F(s)-F(x)-f(x) \sin (s-x)}{s-x}\right| d s=0 .
$$

Thus if $T$ is the Fourier series of $G^{(x)}, D_{s}^{\prime} T_{R}^{\phi}[s] \rightarrow 0$ at $s=x$ for $\phi$ of type $(1,1)$, this means $D_{s}^{\prime} S_{R}[s] \rightarrow f(x) \cos (s-x)$ at $s=x$ where $S$ is the Fourier series of $F$.

\section{REFERENCES}

1. N. K. Bary, On primitive functions and trigonometric series converging almost everywhere, Mat. Sb. 31 (73) (1952). (Russian) 
2. S. Bochner, Summation of derived Fourier series, Ann. of Math. 37 (1936).

3. N. N. Lusin, "The integral and the trigonometric series," Dissertation, 1915, in Collected Works of N. N. Lusin, Moscow, 1953. (Russian)

4. D. Menshov, Sur la convergence uniforme des séries de Fourier, Rec. Math. 11 (1942), 67-96.

5. S. Saks, Theory of the integral, 2nd ed., translated by L. C. Young, Hafner, New York.

VANDERBILT UNIVERSITX,

NASHVILLE, TENNESSEE 